\title{
LA PATRIMONIALISATION DU QUARTIER ANGLO (FRAY BENTOS, URUGUAY) OU LA MÉMOIRE DÉPLACÉE
}

\author{
Ariela Epstein ${ }^{1}$
}

Cette étude c'est initiée dans le cadre d'une recherche collective ayant pour but d'identifier et d'étudier le patrimoine naturel et culturel de la région du sud du fleuve Uruguay $^{2}$. Cette région frontalière est en effet le lieu d'une histoire singulière puisque c'est là que confluaient des milliers de vaches, de l'intérieur des terres, destinées à l'abattage, à la transformation, la mise en boîte et à l'exportation par bateau. Un système rural et industriel composé de fermes d'élevage, d'enclos, d'usines, de frigorifiques et d'installations portuaires couvrait, entre la fin du XIXeme et la moitié du XXeme siècle, l'est de l'Argentine et l'ouest de l'Uruguay. Ce type de bâtiments, ou ce qu'il en reste, sont aujourd'hui réinvestis comme patrimoine historique, symbole d'une époque faste de développement technique et économique, les traces de la révolution industrielle. Chaque «site» est pensé comme une étape sur un parcours touristique plus large, à construire sur les deux rives du fleuve ${ }^{3}$.

En 1863, une entreprise de transformation de viande bovine s'installe à Fray Bentos, Uruguay. La "Liebig Extract of Meat Company", rebaptisée «Anglo del Uruguay » en 1924, marque le début d'un mouvement commercial et industriel de grande ampleur et fait de la petite ville le centre névralgique de la gestion de recours agroalimentaires destinés en particulier à l'Europe. L'extrait de viande en cube et le Corned Beef (viande en conserve) fabriqués à Fray Bentos sont la nourriture principale des soldats alliés durant les deux guerres mondiales. L'usine compte quelques milliers d'ouvriers, dont beaucoup sont de nationalité étrangère. Gérants, techniciens et ouvriers

\footnotetext{
${ }^{1}$ Université Toulouse II Le Mirail, France.

${ }^{2}$ Il s'agit d'un programme ECOS-Sud, intitulé «Analyse et valorisation du patrimoine culturel et naturel du Bas-Uruguay: son rôle dans l'intégration régionale » mené par le laboratoire Géolittomer de l'Université de Nantes et la faculté d'architecture de la Universidad de la República (Uruguay), entre 2009 et 2011.

${ }^{3}$ En plus d'être un attrait touristique, il s'agit aussi de renforcer l'intégration régionale, dans une zone qui fut récemment le centre d'un conflit diplomatique entre l'Argentine et l'Uruguay, lié à l'installation d'une usine de cellulose sur la côte uruguayenne, à quelques kilomètres de Fray Bentos. Sur l'intégration régionale de cette région voir Guibert et al., 2009.

${ }^{4}$ Son premier nom fut Fray Bentos Giebert \& Cie, avec l'entrée de capitaux belges et allemands elle devient Liebig Extract of Meat Company dès 1873.
} 
vivent aux abords de l'usine, dans le quartier Anglo, la première Compagny Town de l'Uruguay. En surplomb, la «casa grande» des gérants puis, en flanc de colline, les petites maisons alignées au toit de tôle pour les ouvriers, le style des habitations diffère selon le statut des habitants. Le quartier possède des clubs de sport et de divertissement - différents pour les anglais et pour les criollos $^{5}$-, une école, une cantine, un centre de santé, le coin des «troperos » ${ }^{6}$, etc. Un système social à petite échelle, sous dépendance anglaise (Lupano, 2009, Boretto, 2000).

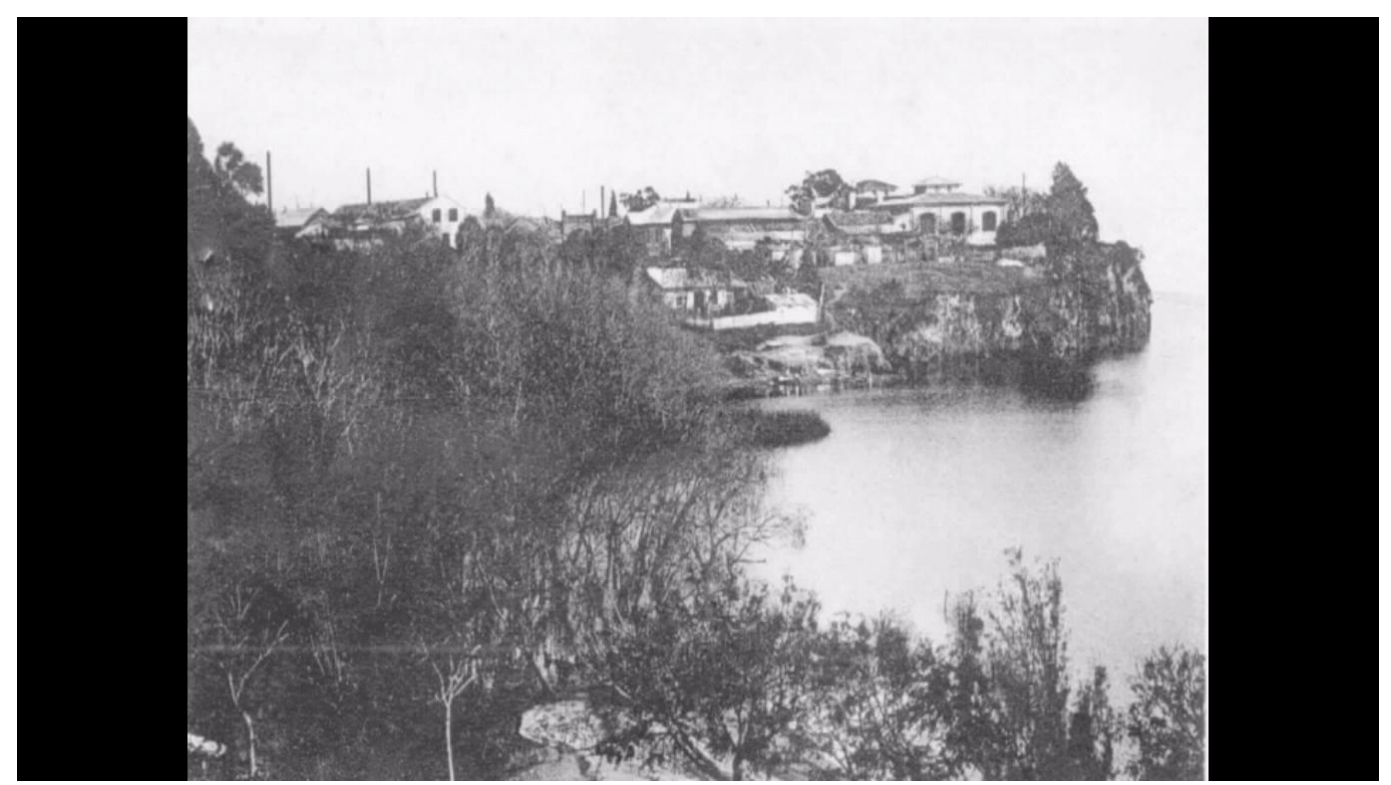

Photo 1: Vue du quartier Anglo, depuis Fray Bentos. Photographie de L1archive «Historiografia de Fray Bentos » de René Boretto Ovalle

\footnotetext{
5 Littéralement les «créoles », mais l'expression désigne plus largement les habitants locaux, en opposition aux étrangers.

${ }^{6}$ Les troperos menaient, à cheval, les troupeaux de bêtes depuis l'intérieur des terres vers l'usine.
} 


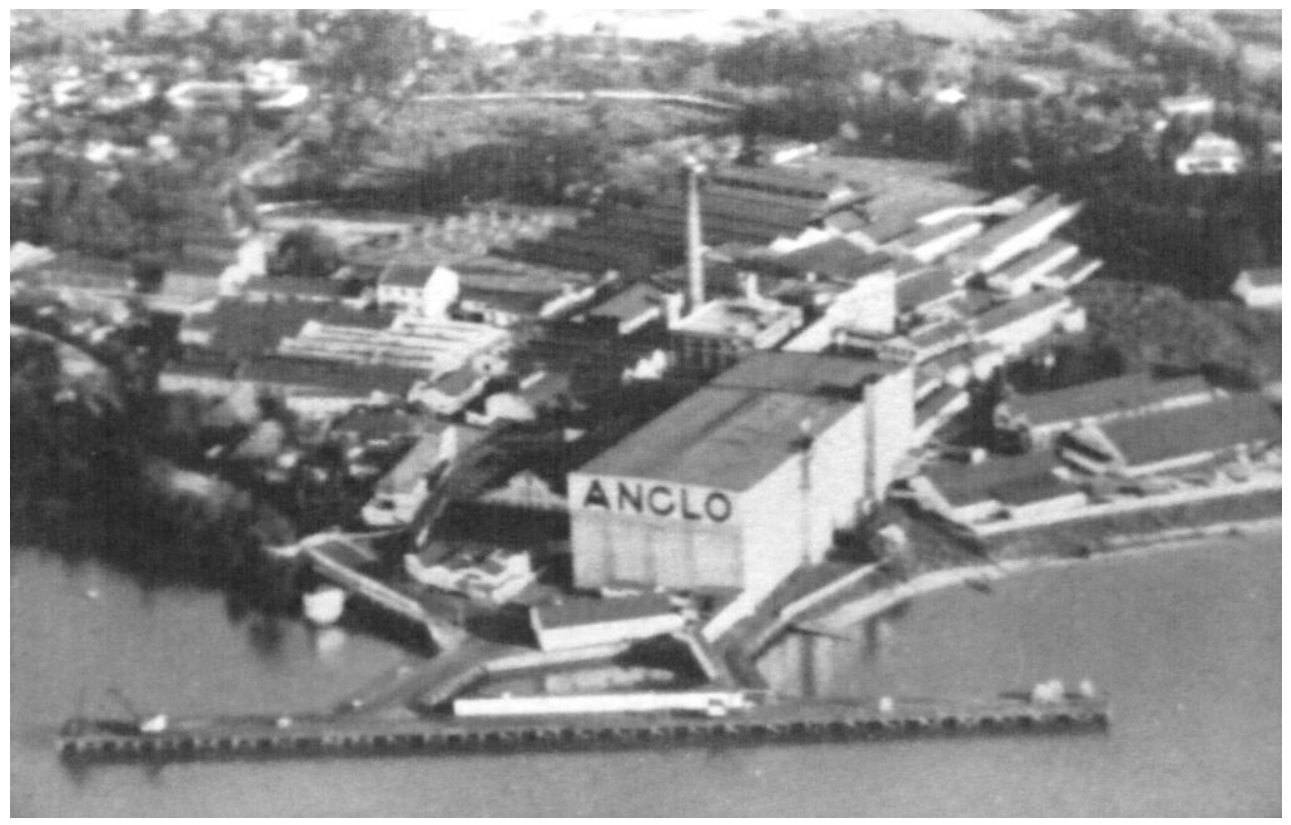

Photo 2 : Vue aérienne de l'usine Anglo del Uruguay. Photographie de l'archive « Historiografia de Fray Bentos » de René Boretto Ovalle.
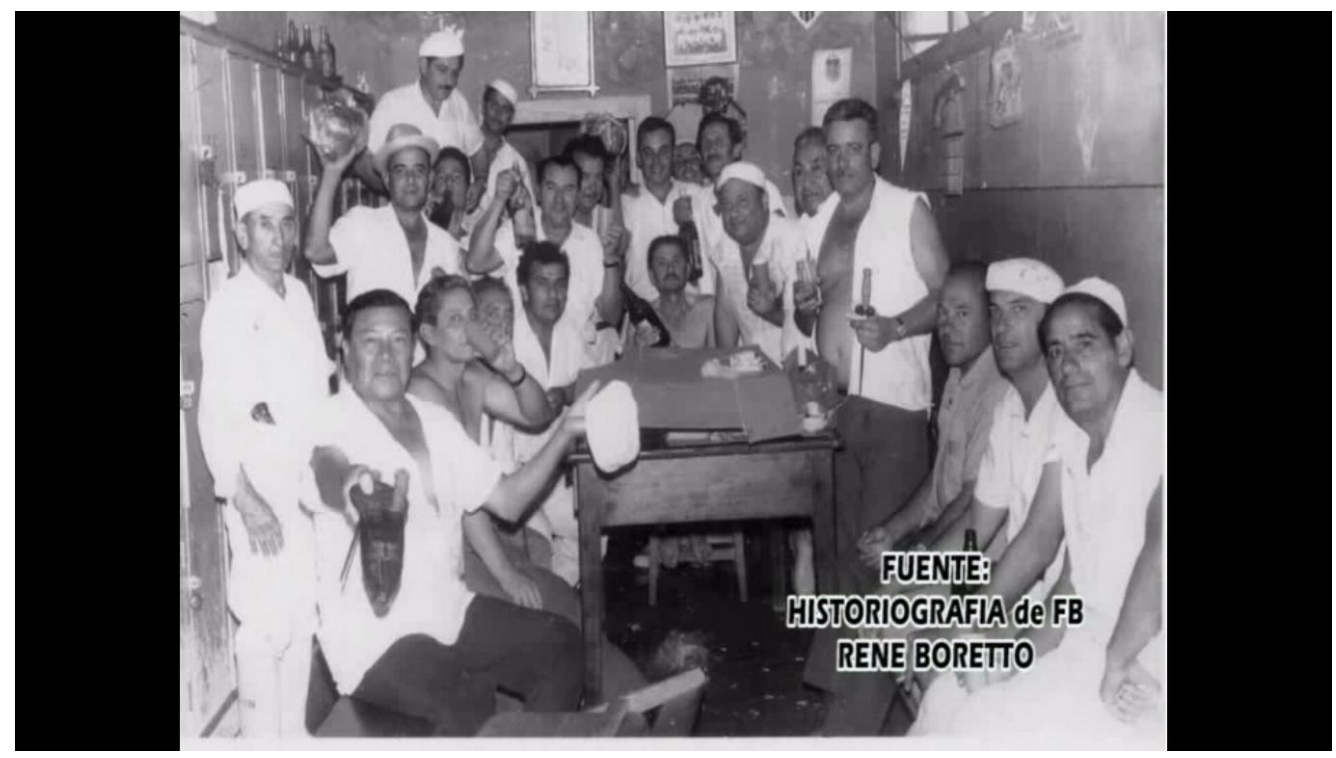

Photo 3 : Ouvriers de l'usine Anglo del Uruguay. Photographie de l'archive « Historiografia de Fray Bentos » de René Boretto Ovalle.

Iluminuras, Porto Alegre, v. 14, n. 33, p. 100-124, jul./dez. 2013 
C'est à Fray Bentos que nous nous installons, pour un mois, Felipe Vargas, photographe, et moi-même. L'équipe de recherche étant composée d'architectes et de géographes, s'attachant aux aspects matériels du patrimoine culturel, nous nous intéressons à ses aspects immatériels, à la mémoire des habitants du quartier. S'agissant d'une mission courte, ce terrain a avant tout une valeur exploratoire.

C'est aussi, pour nous, une expérience de travail en commun dans l'idée d'allier la photographie et l'étude ethnologique, tant au niveau de l'enquête que de sa restitution. Notre objet d'étude nous situe d'emblée un peu en décalage par rapport à la thématique de ce numéro d'Iluminuras, portant sur la mémoire du travail. En effet, le «nœud» de notre enquête réside bien dans une usine fermée, mais les discours receuillis nous ont amenés à déplacer notre regard et à observer les conséquences de cette fermeture sur la vie du quartier ouvrier plutôt que sur l'expérience de l'usine elle-même et sur la relation des hommes au travail. Nous verrons ainsi comment, d'une étude sur le patrimoine local, nous avons finalement pris pour objet le processus de patrimonialisation en cours et ses enjeux pour les habitants du quartier.

\section{Les deux histoires de l'Anglo}

La première image du quartier que nous ayons vue fut une carte géographique que nous a montré l'un des architectes de l'équipe de recherche, à Montevideo, avant que nous ne partions sur le terrain. Sur cette carte de Fray Bentos, le quartier Anglo était zébré de lignes rouges, qui correspondaient dans la légende à une «zone d'habitation irrégulière », formule officielle pour désigner les bidonvilles en Uruguay. Cela suscita notre étonnement, qui fut décuplé lorsque nous sommes arrivés dans ce petit quartier semi-rural, ressemblant plutôt à un village de campagne, formé de petites maisons et de verdure, au bord du fleuve. Cette carte annonçait, avant que nous le comprenions, le paradoxe ou la problématique de cette étude, celui de la «régularité » du statut des habitations, les enjeux de l'habiter dans un monument historique (Fabre, 2010).

Notre première démarche a été de nous familiariser avec le quartier, sa population, les dynamiques qui l'animent. Nous avons réalisé des entretiens avec des référents institutionnels et associatifs du quartier Anglo, ainsi qu'avec des habitants, une vingtaine 
au total. Nous avons alors compris que l'on ne pouvait pas parler d'une mémoire de l'Anglo mais de différents processus mémoriels, tantôt complémentaires, tantôt contradictoires. En effet, il existe plusieurs « récits fondateurs » de ce qu'est le quartier Anglo aujourd'hui, celui des acteurs du patrimoine, le seul légitime dans l'espace public et celui des habitants et des anciens ouvriers de l'Anglo, que l'on pourrait qualifier d'infrapolitiques (Scott, 2009). Il s'est agit pour nous d'interroger ces discours, les manières dont les acteurs en présence s'approprient le passé et le présent du quartier, de se frotter donc, aux questions du patrimoine et de la mémoire.
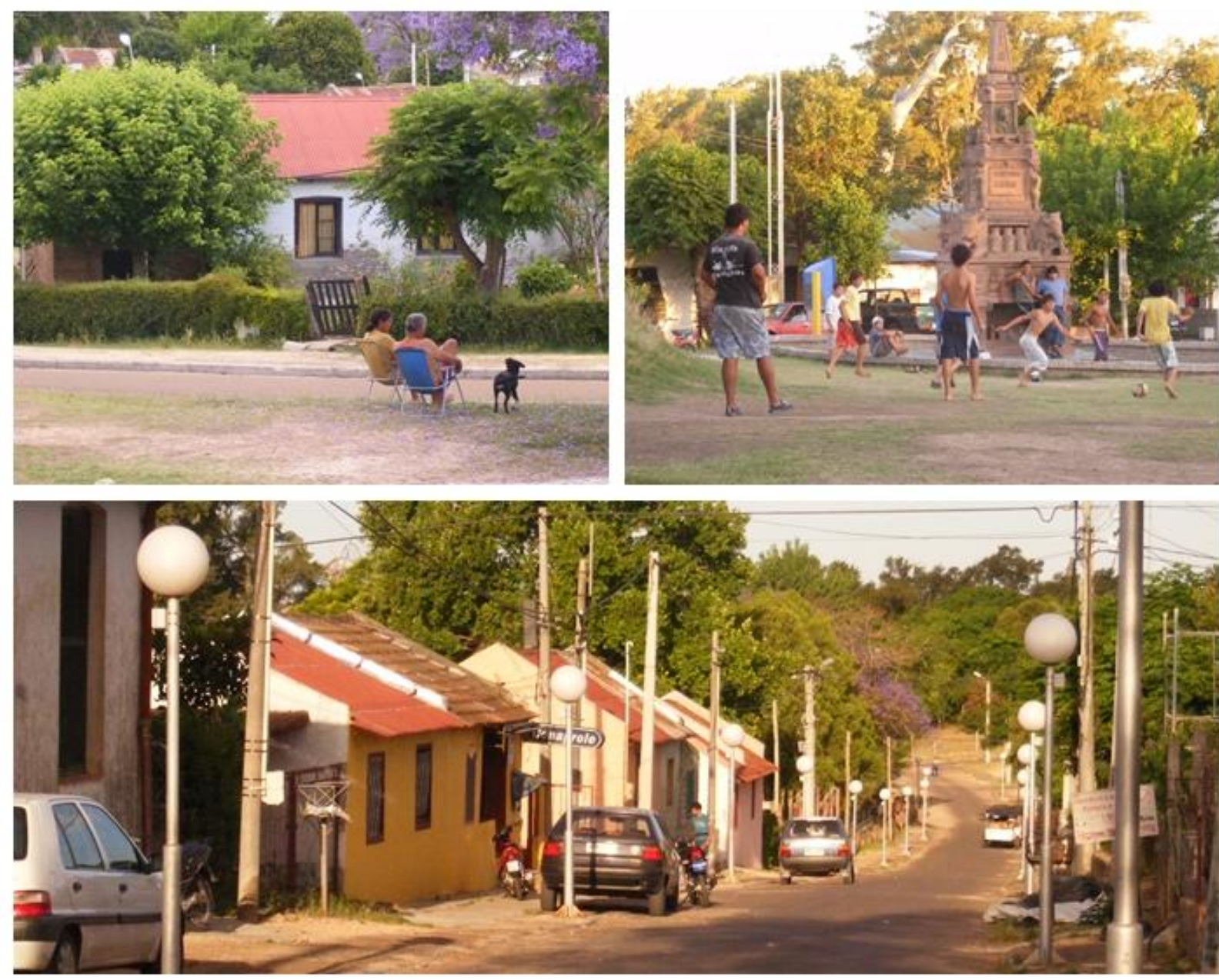

Photo 4, 5 et 6: Vues du quartier Anglo, 2010. Photographie Felipe Vargas.

\section{a) La fabrique du patrimoine}

Dans les années 1970, après un siècle d'activité, l'usine Anglo del Uruguay ferme

Iluminuras, Porto Alegre, v. 14, n. 33, p. 100-124, jul./dez. 2013 
progressivement. Les bâtiments industriels, tout comme le quartier, deviennent propriété de la mairie. À la fin des années 1980, le maire de la ville, Mario Carminatti lance une dynamique de récupération et de mise en valeur du site. L'usine, le port fluvial et une partie du quartier ouvrier sont déclarés «Monument historique de la Nation » en juin 1987. Le «Musée de la Révolution Industrielle » ${ }^{7}$ ouvre en 1994 dans un des bâtiments de l'usine. La Commission de gestion du système patrimonial industriel Anglo travaillant sous la tutelle de la mairie, de la Commission du Patrimoine Culturel de la Nation (attachée au ministère de l'éducation et de la culture), du ministère du logement et de l'aménagement du territoire-, développe depuis une dizaine d'années un programme de réhabilitation et de valorisation du patrimoine industriel. En 2008, l'équipe obtient l'amplification du site patrimonial et en 2010, elle soumet à l'UNESCO le projet «Paysage culturel Fray Bentos», en vue de l'obtention du label de «patrimoine mondial de l'humanité ». Les chercheurs et experts formulent des préconisations en termes de protection et de mise en valeur ${ }^{8}$, de réintroduction d'activités productives ${ }^{9}$, d'aménagement urbain et d'une manière générale, de tout ce qui concerne l'usine et le quartier. Le «paysage culturel » est mis en avant comme le résultat de la main de l'homme sur les merveilles de la nature, une histoire du développement qui se voudrait exemplaire.

Dans cette même perspective, les personnels de la Commission du patrimoine culturel de la Nation mènent actuellement deux projets simultanés dans le quartier (Chagas et al., à paraître). Le premier, encore en préparation, vise à évaluer la possibilité d'impliquer les habitants du quartier dans le projet de labellisation. Le second est la constitution d'une «archive des mémoires de l'Anglo » composée essentiellement de témoignages d'anciens ouvriers. Des entretiens sont réalisés, qui touchent aux

\footnotetext{
${ }^{7}$ Ce musée fut fondé par René Boretto Ovalle, historien uruguayen. Il fut le directeur du musée pendant de nombreuses années.

${ }^{8}$ Voir notamment les différents articles publiés sur les sites institutionnels de la région du Río Negro et de la Commission du Patrimoine de la Nation, dont, par exemple : Ménanteau et Boretto Ovalle, 2005 ; Boretto Ovalle et Burgueño, 2009 ; et Rey Ashfield, 2009.

${ }^{9}$ La zone industrielle de l'Anglo est immense et possède une grande quantité de hangars et de locaux vides. Le Comité de gestion du patrimoine favorise donc l'installation d'entreprises, pour redonner vie au site et pour assurer une partie de son entretien. De nombreuses petites entreprises se sont installées, mais rarement de façon pérenne. Il y en avait 4 ou 5 en 2010, dont un laboratoire argentin de fertilisants, un atelier de poterie, un traiteur.
} 
différentes expériences et mémoires du travail à l'usine, mais aussi à la vie dans le quartier et aux liens existants entre ces deux espaces ${ }^{10}$. Les employés du musée, formés et encadrés par les ethnologues de la mission du patrimoine, sont en charge du recueil et de la compilation de ces archives qui seront ensuite disponibles sur un site multimédia, consultable sur internet. D'une manière générale, la mémoire portée par les acteurs institutionnels, visible aujourd'hui à travers le musée et quelques rares publications (Boretto Ovalle, 1993, 2000 ; Campodonico, 2000 ; Taks, 2000), renvoie à l'histoire de l'usine en fonctionnement, à l'organisation du travail et aux innovations techniques qui y ont eu lieu. C'est aussi la mémoire d'un monde d'abondance - une des anecdotes souvent racontée est que chaque travailleur de l'usine avait droit à 2 kilogrammes de viande par jour- et celle d'une petite société, à part, à l'anglaise, réglée par la vie de la fabrique, et marquée par l'immigration, puisque les ouvriers étaient originaires de 120 nationalités différentes.
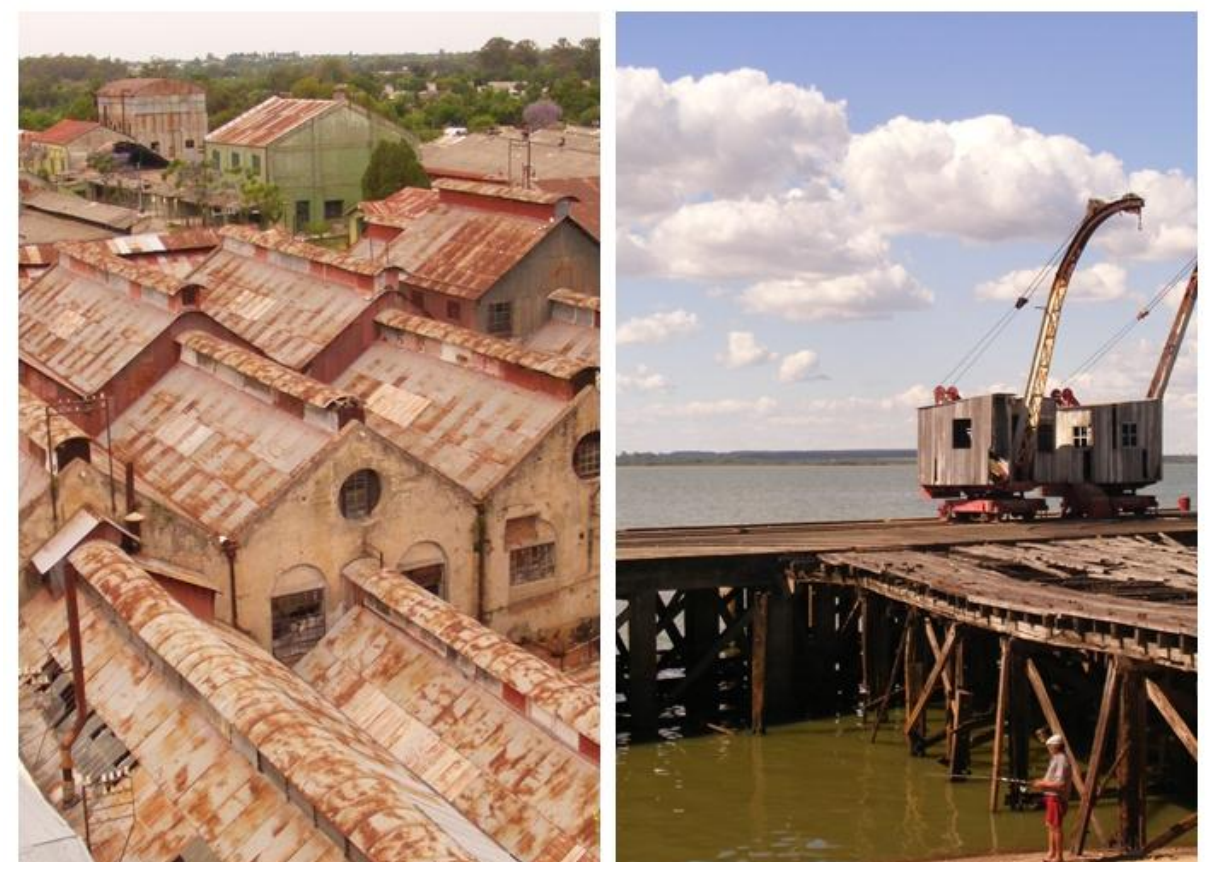

Photo 7 et 8 : Vues de l'usine en 2010. Photographies Felipe Vargas.

Les habitants que nous avons interrogés semblent disposés à accompagner le processus de labellisation patrimonial en cours, qui leur paraît plutôt positif. Leur sentiment d'appartenance au quartier est fort. Ils en connaissent l'histoire et se disent

\footnotetext{
${ }^{10}$ Cette enquête étant en cours de réalisation, je n'ai connaissance d'aucun résultat de celle-ci.
} 
prêts, par exemple, à servir de guides pour informer les touristes de passage. À l'école du quartier, ouverte en 1884, des institutrices mènent des projets de recueil de témoignages avec les élèves, et travaillent avec eux sur l'histoire industrielle. Enfin, la plupart des institutions datant de l'époque de l'usine sont encore en activité : le club de foot, celui de jeux de boules, le club social « la Estrella », la crèche, le centre médical, etc. Elles assurent les mêmes fonctions sociales qu'avant. La sociedad nativista el Rincón, club rural installé depuis une dizaine d'années à la sortie du quartier Anglo, est dédiée aux traditions équestres locales (concours de rodéo, dressage, etc). Elle commémore également la grande époque industrielle, et ses prolongements ruraux, en redonnant vie à ce qui fut « le coin des troperos ». On y maintient les traditions locales, comme les peñas musicales ${ }^{11}$ ou la confection de l'asado con cuero. Enfin, les habitants se prêtent avec plaisir au jeu des commémorations, telles que le défilé lors des cent cinquante ans de la fondation de Fray Bentos, où ils représentaient fièrement leur quartier et son histoire.

\footnotetext{
${ }^{11}$ Les peñas sont des soirées musicales où artistes et public interprètent des chansons traditionnelles.
} 


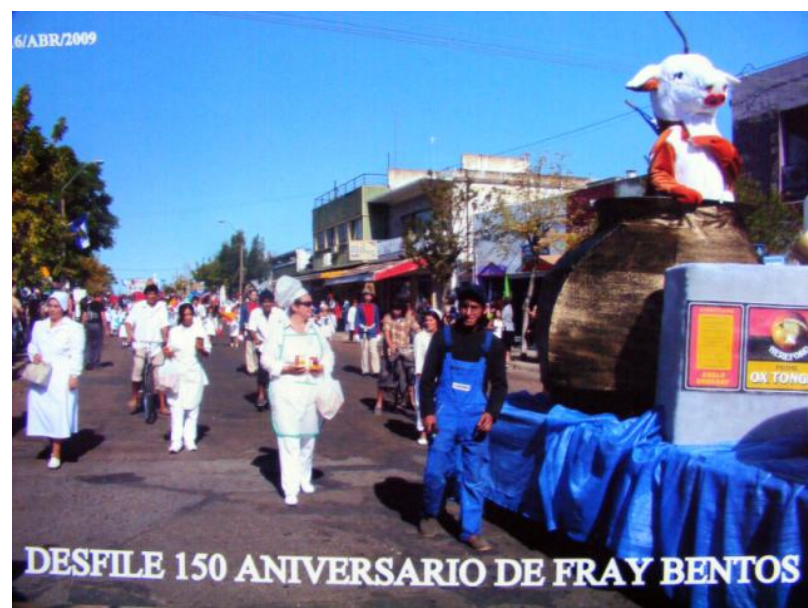

Photo 9 : Souvenir du défilé des 150 de Fray bentos. Les habitants du quartiers sont déguisés en ouvriers de

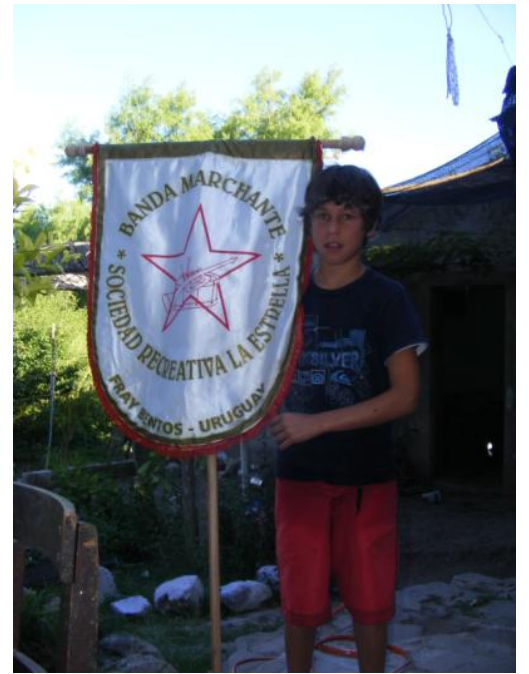

Photo 10: Um jeune habitant porte l'étendard de l'orchestre Du quartier, reforme Durant quelques années. Photographie Felipe Vargas.

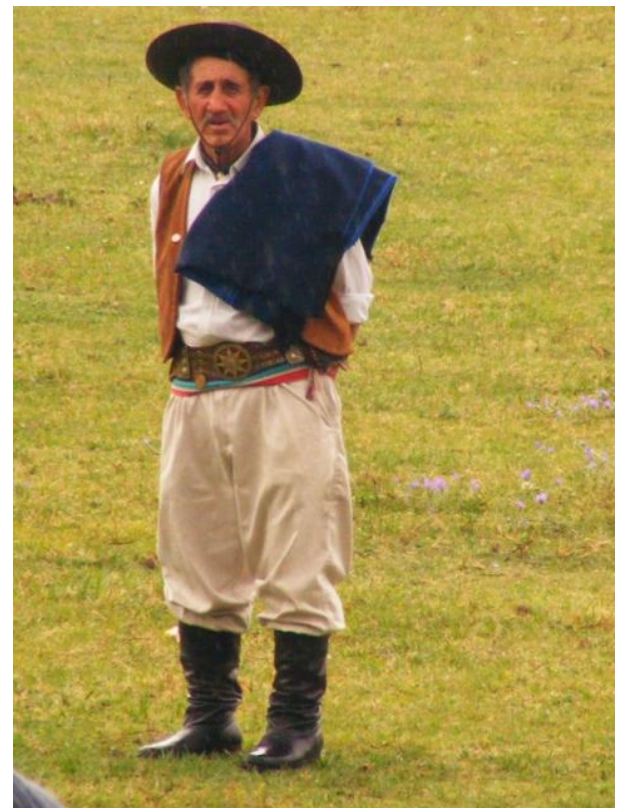

Photo 12: Un gaucho, lors des fêtes criollas de la Sociedad nativista el Rincón. Photographie Felipe Vargas.

Photographie Felipe Vargas

Acteurs institutionnels et habitants semblent ainsi s'accorder sur l'existence d'un passé heureux, singulier, marqué par les différentes activités liées à l'usine, mais aussi à la qualité de vie qu'offrait le quartier. Un quartier qui, par son histoire, son architecture et sa localisation géographique, a développé un fort sentiment identitaire. "Acá es un 
paraíso » nous dit une habitante :

-Teníamos todo exclusivo. Acá le decimos la República del Anglo, como si fuera un mundo aparte! Del puente para allá es Fray Bentos, acá es el Anglo, ¿viste? Siempre fuimos exclusivos. [...]La verdad que somos un pueblo aparte.

-Con una identidad fuerte, ¿no?

-Ah si, si. Porque los que somos realmente del Anglo, peliamos por el Anglo, ¿viste?

Il existe donc, à l'Anglo, une mémoire collective qui fait consensus et fabrique de l'identité, une mémoire propre à devenir du patrimoine. Mais son énonciation pose d'emblée le cœur du problème, «être vraiment de l'Anglo » n'est pas vraiment une évidence, la «lutte» semble en être une condition. Les habitants de l'Anglo sont supposés être les anciens ouvriers de l'usine et leur famille mais la réalité est, bien sûr, plus complexe.

\section{b) Récits d'une non-histoire}

Dans la vingtaine d'entretiens réalisés avec les habitants de l'Anglo, lorsque nous abordons la vie et l'histoire du quartier, le temps de l'usine en marche n'est généralement pas ou peu évoqué. Les habitants font commencer leur récit après la fermeture. Dans les années 1970, c'est-à-dire durant la dictature militaire (1973-1985), l'usine ferme, peu à peu, par secteurs de production. Au début des années 1980, tous les habitants reçoivent une lettre notifiant qu'ils doivent quitter le quartier, au plus vite et de façon définitive. Les raisons de cette «expulsion » et le récit des événements qui l'entourent sont confus. La plupart accuse «la dictature » ou « les militaires », qui auraient eu l'intention de raser le quartier pour construire une base et un port. On invoque aussi parfois «Saudico », un groupe ou une famille qui avait racheté l'usine en faillite en 1983 ou $4^{12}$ et aurait également «négocié » le quartier, mais vide. Il n'existe à notre connaissance aucun écrit institutionnel, scientifique ou journalistique, faisant écho à cette période mais il s'agit, comme nous l'avons signalé, d'un travail préliminaire.

Les habitants s'accordent tout de même sur quelques grandes lignes de l'histoire. Suite à la lettre leur demandant de quitter les lieux, deux complexes d'habitations, le

\footnotetext{
${ }^{12}$ Les dates sont floues, tout ce que nous relatons ici sont des données d'entretiens.
} 
JC6 et le JC7, sont construits à Fray Bentos, pour les ex habitants de l'Anglo. Des camions militaires transportent les habitants et leurs biens vers leur nouvelle résidence, qui leur est donnée à titre gratuit, ou avec peu de charges, selon les revenus de chacun. Le quartier se vide peu à peu. Cependant, quarante six familles, sur environ deux cent, décident de «résister» et de ne pas partir. Les quelques personnes représentant ces familles, avec qui nous nous sommes entretenus, racontent qu'il y eut alors une période difficile. Si aucune expulsion de force n'est mise en œuvre, la pression et les tentatives de dissuasion sont multiples et constantes. L'eau et l'électricité, fournies à l'époque par l'usine, ne sont dispensées que quelques heures par jour, en début de soirée. Des contrôles sont systématiquement mis en place à chaque entrée et sortie du quartier, le droit de réunion est proscrit. Les personnes soupçonnées d'avoir des activités politiques sont molestées, interrogées et leurs maisons fouillées.

Un ensemble de 35 maisons, un sous-quartier appelé «La Pandilla» est finalement démoli. Tous racontent la peur permanente, le bruit des pillages des maisons désertées, pendant la nuit, et les conditions de vie précaires dans ce quartier qui avait été la gloire de la région, le scandale de devoir s'éclairer à la bougie, là où avait brillé la première ampoule électrique de tout le pays.

En 1987 l'usine et le quartier sont déclarés Patrimoine de la Nation, et sont donc protégés. Selon une habitante, cette qualification patrimoniale est l'aboutissement du travail de quelques familles résistantes, accompagnées par l'architecte Mariano Arana ${ }^{13}$, et elle avait pour but explicite d'arrêter la destruction du quartier. Si elle évoque l'existence de nombreux documents conservés par des habitants ${ }^{14}$, aucune autre mention ne nous est faite de cette première étape d'un processus qui, nous l'avons vu, à ensuite été pris en main par les institutions. Les maisons vides sont peu à peu occupées, de manière spontanée et irrégulière, par des familles venant de Fray Bentos ou de la région. Avec la mise en place d'un programme de réaménagement urbain, le PIAI ${ }^{15}$, un

\footnotetext{
${ }^{13}$ Mariano Arana, membre du Frente Amplio fut maire de Montevideo entre 1994 et 2005.

${ }^{14}$ Ayant su cela lors du dernier entretien, nous n'avons malheureusement pas pu nous enquérir de ces documents.

15 PIAI : Programa de Integración de Asentamientos Irregulares. Les programmes PIAI dépendent du ministère du logement et de l'aménagement du territoire. Il est destiné à régulariser les zones d'habitations irrégulières, c'est à dire le plus souvent les bidonvilles et vise à les mettre aux normes sanitaires. À l'Anglo il s'est agit de travaux de réaménagement des espaces publics, des canalisations, des circuits électriques, la construction de maisons pour éviter le surpeuplement, etc.
} 
recensement réalisé en 1992 officialise la résidence de tous les habitants, quelque soit leur ancienneté sur place.

Ainsi, aujourd'hui, la population de l'Anglo donne corps à trois catégories d'habitants, porteuses de différents discours. La première rassemble les 46 familles résistantes et que l'on nomme là-bas les «familles historiques » ou « originaires ». La deuxième se compose des «nouvelles» familles de l'Anglo, qui se sont installées depuis la fin des années 1980, lorsque les militaires ont quitté le quartier. Après une période de va-et-vient dans les maisons, de divisions des bâtiments et de transactions illégales, de nombreuses familles se sont stabilisées et sont désormais « du quartier ». La dernière catégorie d'habitants ne se trouve pas à l'Anglo, elle réunit les familles ouvrières relogées dans les deux complexes d'habitations construits pendant la dictature militaire.

Nous nous sommes rendus dans l'un des deux. Le JC6 est un petit groupe d'immeubles à la sortie de Fray Bentos, huit bâtiments de quatre étages, aujourd'hui vétustes et insalubres. Nous décidons donc de délocaliser temporairement notre enquête, considérant que c'est là aussi que se trouvent les anciens ouvriers et leur familles, dont les acteurs du patrimoine souhaitent sauvegarder la mémoire.

\section{Ethnographie d'une mise en patrimoine}

Les entretiens nous ont fait prendre des chemins inattendus et nous nous interrogeons peu à peu sur l'intérêt, dans ce contexte, de «collecter » du patrimoine culturel. Il s'agit plutôt de comprendre et de traduire les discours engendrés par les processus actuels, leurs tensions et leurs enjeux, c'est-à-dire de prendre le «patrimoine en-train-de-se-faire » pour objet ethnologique (Tornatore, 2004). Nous nous arrêterons donc, d'une part, sur les façons dont les habitants se situent aujourd'hui face au quartier Anglo et aux dynamiques qui l'animent, et proposerons, de l'autre, quelques réflexions sur la mémoire de ces lieux, érigée en patrimoine ou passée sous silence.

\section{a) Repositionnements actuels}


Cette histoire d'expulsions et de résistances, fortement politique, a bien entendu des conséquences sur les manières dont chacun investit aujourd'hui le quartier, et dans les significations qu'il lui confère. En observant les familles de l'Anglo et leurs différentes formes d'implications dans la vie du quartier, en nous approchant du Comité de quartier, des différentes associations, nous avons commencé à observer des pratiques quotidiennes et des dynamiques collectives qui le font vivre, et y impriment une «identité ». Créé pendant la dictature, le Comité de quartier a pendant un temps cessé toute activité, puis s'est reformé avec la mise en place du programme PIAI. Il réunit aujourd'hui quatre ou cinq membres actifs, descendants des familles historiques ou arrivés dans le quartier dans les années 1990.
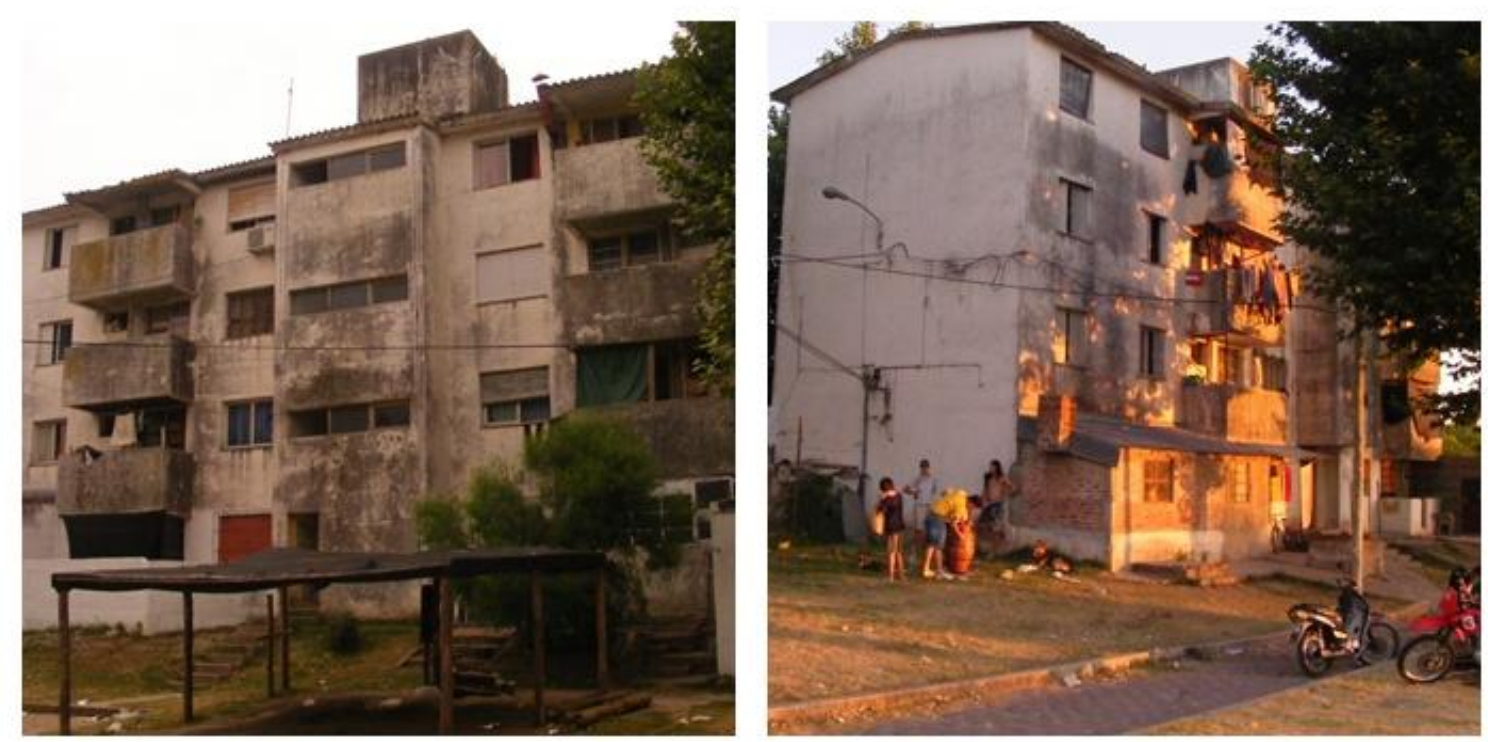

Photo 13 et 14: Vues du quartier JC6. Photographie Felipe Vargas

Le programme PIAI occupe en fait une grande partie des entretiens. Tout ce passe comme s'il venait soulever à nouveau les questions non réglées du passé. Il fallait, nous dit-on, l'accord des habitants pour mettre en œuvre les travaux. Cet accord a été obtenu suite à quelques réunions, mais une de ses clauses n'a pas encore été soldée. En effet, le programme doit aboutir à la délivrance de titres de propriété aux habitants pour la maison qu'ils occupent, mais cette dernière étape est sans cesse repoussée pour des raisons administratives liées, de fait, à la valeur patrimoniale des bâtiments. Propriétés privées de l'usine puis de la mairie, les habitations sont devenues patrimoines. Selon les 
législations, ce type de monument peut être soit « relié » à ses habitants, qui deviennent eux-même «historiques »soit, au contraire, le bien étant commun, on évite qu'il ne devienne la propriété d'un individu. On peut penser qu'à l'Anglo c'est l'indécision des pouvoirs locaux quant aux choix concernant les maisons qui pose problème, ce cas faisant en quelque sorte jurisprudence 16 .

D 'une manière générale, les habitants se disent favorables aux deux grands projets, urbanistique et patrimonial, mais quelques-uns réclament avec force leur titre de propriété et donc l'assurance qu'ils ne seront plus jamais contraints de partir. La question de ces papiers officiels fixe et révèle une histoire douloureuse, la colère et les larmes viennent vite pendant les entretiens. On nous dit, à plusieurs reprises, que certains ont lutté toute leur vie et sont morts sans avoir eu ce papier. Une ancienne habitante raconte :

No me parecía justo, es que en ese momento había personas que ya les quedaba poco tiempo de vida, porque eso fue en el 2006 que se firmó, estamos en el 2010 y ya no están...ni siquiera llegaron a disfrutar de su casa. El último fue Bordoli que me decía "seguí peleando [ silence, elle pleure] seguí peleando que esto no puede quedar así'.

Les anciens habitants avec qui nous avons parlé montrent un fort attachement au quartier et ils entendent être acteurs des décisions qui le concernent. Il s'y impliquent à titre individuel et en participant bénévolement aux activités du centre de santé, de l'école ou des différentes associations récréatives. On voit également qu'il y règne, même si c'est de façon ténue et discrète, une tension liée à la légitimité de la présence et de la place de chacun. En plus de la demande des titres de propriété, celle-ci s'exprime à travers des dénonciations d'injustice dans les bénéfices octroyés par le programme $\mathrm{PIAI}^{17}$, dans la frustration générée par un projet de construction d'une coopérative d'habitat lancé par les habitants et refusé par les pouvoirs locaux, d'une requête des

\footnotetext{
16 Cette explication n'est qu'une hypothèse. Les seules raisons qui nous aient été données sur l'ajournement de la délivrance des titres de propriété sont liées à la complexité du découpage du cadastre.

${ }^{17}$ Le programme octroie des services, comme des travaux d'amélioration des maisons, entièrement gratuit pour les familles les plus pauvres. De nouvelles maisons ont été construites, respectant le modèle architectural des anciennes, pour y reloger les familles qui vivaient en surnombre. Ces familles bénéficiaires sont de fait, pour la plupart, des habitants installés dans les années 1990. Les habitants « historiques », généralement en situation moins précaires, ont du payer pour les travaux qui les concernaient.
} 
« anciens » pour reconstruire le quartier de la Pandilla, également écartée, mais surtout dans la relation avec les «nouveaux » habitants. On se trouve, en effet, face à un double discours, qui souligne à la fois une intégration réussie et un manque d'investissement général. On nous parle à plusieurs reprises du «travail d'intégration » mené auprès de ceux que l'on appelait à un moment «los agregados ». La distinction, très forte dans un premier temps, aurait fini par s'estomper. Ainsi, une institutrice de l'école, y travaillant depuis une vingtaine d'années, explique :

Cuando llegué costó que la gente tome consciencia, porque la gente que está viviendo acá no es la de antes. Son pocos los que quedaron de la época del frigorífico. La gente vino de otros barrios de Fray Bentos, no tenía sentimiento de pertenencia al barrio. Todo lo que se les daba lo vendían, no lo cuidaban, rompían... No pertenecían, venían porque las casas estaban vacías.

Elle considère que les programmes de requalification et de patrimonialisation favorisent également cette intégration au quartier :

Va a servir para los chiquilines, porque antes, en la época brava, cuando se llenaron las casas, uno hablaba de la Escuela 3 y era como : "Uy, la Escuela 3, que horrible". Pero ahora ha cambiado. No hay problema de conducta, con los padres todo bien, estamos cerca de los padres [...] Ya no somos los indios del Anglo, es otra generación, ya no es como antes.

Si le quartier s'est aujourd'hui défait de sa «mauvaise réputation », les membres du Comité de quartier comme les «habitants historiques», regrettent pourtant le manque de mobilisation et d'implication des « nouveaux » habitants dans la vie de celuici. Par exemple, s'ils ont généralement participé aux premières rencontres publiques organisées par les représentant du programme PIAI et de la Commission de gestion du système patrimonial, presque tous s'en sont rapidement éloignés.

Pour les familles arrivées dans les années 1990, le processus de patrimonialisation et le devenir de l'usine n'est pas une question importante, celle des titres de propriété ne semble pas non plus être une préoccupation. Ils attendent que des décisions claires soient exprimées car de celles-ci dépendent les autorisations pour transformer leur habitat -ouvrir une fenêtre supplémentaire dans la maison, construire une remise dans le jardin, etc. - mais sachant que les familles avec enfants ne peuvent pas, légalement, être expulsées de leur domicile, la propriété ne signifie, pour beaucoup, que des coûts 
fiscaux supplémentaires.

Les enjeux sont donc différents selon le statut et l'histoire de chacun dans le quartier, les «anciens » et quelques autres habitants ralliés à leur cause revendiquent en toute occasion leur légitimité et leurs positions dans les décisions concernant le quartier. Les autres n'en ont en quelque sorte pas besoin. Finalement, la «prise en main » par des acteurs extérieurs au quartier, chargés de « régulariser » les habitants, spécifiant leurs droits et leurs devoirs, a réveillé un sentiment de dépossession et engendré la réclamation d'une reconnaissance particulière.

Au JC6, nous avons rencontré trois anciens habitants de l'Anglo. Leur version de l'histoire est sensiblement la même, jusqu'à leur départ. Puis ils sont restés là, et il n'y a pas grand chose d'autre à dire. La vie actuelle du quartier Anglo ne les concerne plus et leur discours est globalement résigné, passif. Les habitants du JC6 se plaignent des conditions sanitaires dans lesquelles ils vivent, leurs récits concernent l'incompréhension des raisons pour lesquelles ils sont là et le fait que, n'ayant pas été démolies, leurs anciennes maisons aient finalement été occupées par d'autres. «Nos trajeron acá », ou «la dictadura nos puso acá » sont des formules qui reviennent souvent, exprimant à la fois une contrainte et un coupable plus que flou. Le déplacement forcé a opéré une rupture si forte que leur discours rappelle ceux des exilés, des expatriés, alors même qu'ils ne se trouvent qu'à deux kilomètres de leur terre.

La question du retour est évoquée à de nombreuses reprises, comme un idéal que l'on ne pense pas vraiment réalisable et pour lequel aucune démarche concrète n'a été mise en œuvre, «si me dan una casa, yo vuelvo ». Les habitants regrettent leur pays perdu ${ }^{18}$, où ils faisaient partie d'une grande famille, la tonalité dominante des entretiens est nostalgique, un sentiment qui, selon Breviglieri « rendrait tangible une expression résiduelle, dénuée de substance politique, et non pas une parole résistante. » (Breviglieri, 2010) L'amertume, parfois l'ironie, imprègne les discours quand les langues se délient. Si l'histoire des résistants de l'Anglo est peu connue, la leur l'est encore moins, ils ont été littéralement écartés de l'histoire officielle.

\footnotetext{
${ }^{18}$ Pour une approche anthropologique de la nostalgie, voir notamment le dossier « Pays perdus, pays imaginés » coordonné par Valérie Feschet et Cyril Isnart (Feschet, Isnart, 2013).
} 


\title{
b) Un passé peut en cacher un autre
}

\begin{abstract}
Si la mise en patrimoine, notamment muséographique, apparaît généralement comme une forme spatialisée d'institutionnalisation d'une mémoire valorisée, signe d'un certain rapport des sociétés modernes à l'histoire, elle crée un type paradoxal de muséification du social, situé entre mémoire et oblitération, qui semble viser à enfermer, figer et soustraire à la visibilité obligatoire les emblèmes encombrants d'un passé ayant perdu toute légitimité.(Losonczy, 2006: 98)
\end{abstract}

Plusieurs éléments dans cette phrase empruntée à A. M. Losonscy, font écho aux problématiques qui nous intéressent ici. La définition de «mise en patrimoine », tout d'abord, en tant que processus institutionnel et comme forme de valorisation d'un passé heureux, bon ou revendicable ${ }^{19}$. Ensuite, le paradoxe que créent les institutions patrimoniales en conformant, (dé)limitant et faisant parfois disparaître certains discours, ou certaines mémoires. À l'Anglo, les récits des expulsions, de la résistance et de «l'exil », sont complémentaires de la mémoire industrielle mise en avant par les institutions et les intellectuels, elles en sont la suite. Mais, révélant la mort peu glorieuse de l'usine et l'échec d'un modèle économique fondé sur la dépendance, l'histoire des dominés devient «encombrante ». Cela nous mène à nous demander quelles mémoires deviennent du patrimoine et surtout ce que deviennent les autres, qui n'entrent pas dans ce cadre. Le patrimoine-en-train-de-se-faire (Tornatore, 2004) ne se faisant pas tout seul, il convient de s'arrêter non seulement ce qui devient du patrimoine, mais sur qui le désigne et aux yeux de qui celui-ci est, ou non, légitime. La perspective de l'observation d'un processus de patrimonialisation «s'intéresse moins aux objets ou aux faits qu'à leur sociologie ; [...] Elle éclaire sur les «cristallisations » de la mémoire, de l'histoire et de l'identité, et les politisations locales, à faible ou moyenne portée, qui en sont issues. » (Tornatore, 2004) Cela implique notamment la question de ce que déclenche la patrimonialisation d'un lieu pour ses habitants, les sentiments -politiques ou non- et les rapports au passé que celle-ci engendre.

Les récits des habitants, nous l'avons dit, commencent avec la fermeture de l'usine. Cette fermeture, et aussi, ou surtout, les expulsions qui lui ont succédé, apparaissent comme un second moment fondateur dans l'histoire du quartier, le premier

\footnotetext{
${ }^{19}$ C'est du moins la conception la plus courante du patrimoine, telle qu'elle est portée notamment par l'UNESCO.
}

Iluminuras, Porto Alegre, v. 14, n. 33, p. 100-124, jul./dez. 2013 
étant la construction du site. Il s'agit donc d'un événement, une rupture suivie d'une réorganisation du monde, qui scinde le passé en un avant et un après (Bensa et Fassin, 2002). Les récits de cette époque, particulièrement flous, en font un événement étrange, dont il ne reste que du vécu, des souvenirs, et qu'aucune trace écrite ni aucun commentaire officiel ne vient valider. Il constitue le contraire d'un événement-montre (Nora, 1972), un non-événement, dénié et donc difficilement appropriable. Par ailleurs, tout se passe comme si, avec le déplacement forcé, ou sa menace, s'était opéré un déplacement de la mémoire des habitants et de leurs centres d'intérêts. Le quartier, les maisons, prennent bien plus de place dans les discours, que le travail à l'usine ou la disparition de celui-ci ${ }^{20}$.

À l'Anglo comme au JC6, d'un entretien à l'autre, nous tentons de comprendre les logiques d'une mémoire collective, à partir de bribes de mémoires individuelles; une mémoire qui mobilise le plus souvent une rhétorique de victime. Les habitants expriment la violence symbolique vécue dans les années 1970 et 80, en la raccrochant aux processus actuels, sous des formes de politisation ou au contraire par la passivité et l'indifférence.

De notre point de vue, cet événement, cette seconde histoire de l'Anglo, réclame tout d'abord une étude historique basée sur la recherche de documents, visant à reconstituer les faits « réels », objectifs. D'autre part, une « intervention ethnologique » (Tornatore, 2007) capable de répondre à la demande sociale de raconter et peut-être d'apaiser l'histoire d'après l'usine semble avoir toute sa place. Observant le présent affecté par les ruptures du passé, cette perspective fait suite aux nombreux travaux anthropologiques réalisés, en France notamment, autour de fermetures d'usines et de patrimoines industriels ( Rautenberg et Faraut, 1994 ; Daumas, 2006 ; Tornatore, 2006 ; Veschambre, 2008), qui considèrent les dispositifs patrimoniaux en termes de deuil et visent à «décrire toutes les actions participant au règlement symbolique de la perte, dans le territoire qui en est défini : en d'autres termes, une « tentative d'épuisement de l'espace de la mémoire » » des lieux (Tornatore, 2006: 2).

\footnotetext{
${ }^{20}$ De nombreux habitants de l'Anglo sont retraités. Il y a aussi beaucoup de fonctionnaires et de personnes vivant de «changas », des activités irrégulières, le plus souvent informelles. La pauvreté et le chômage y sont présents mais pas plus ni moins que dans le reste de la région.
} 
La mémoire en jeu à l'Anglo, patrimoniale ou non, est bien celle qui relie et sépare les hommes et les espaces de vie, l'attachement et le déracinement en sont les deux revers.

Par ailleurs, l'absence d'écrits ou les réponses évasives des acteurs institutionnels par rapport à la seconde histoire de l'Anglo n'est pas, à mon sens, un déni volontaire et politique. Elle reflète plutôt un désintérêt, une histoire qui ne les concernent pas. On peut se demander, aussi, pourquoi revenir sur les temps de dictature et de «décadence » du quartier, au moment où les dynamiques en cours veulent «aller de l'avant » ? Ces interrogations ont d'autant plus de sens qu'en Amérique latine, la mémoire et la résolution du passé dictatorial est éminemment politique et incandescente d'actualité. À l'Anglo, ces questions se posent de manière particulière. Les habitants se décrivent comme des victimes de la dictature, mais ne réclament ni vengeance ni même identification des responsables. On peut, en revanche, envisager avec D. Fassin et R. Rechtman, leur position en terme de traumatisme et donc de réparations symboliques, de témoignages et de construction de preuves comme ressources sociales pour la revendication de leurs droits (Fassin et Rechtman, 2007). On est loin des grandes réclamations de justice qui agitent le continent, mais dans l'énonciation d'une histoire «petite », discrète et néanmoins en quête de légitimité; les habitants veulent la reconnaissance symbolique de ce qui est leur histoire et la sécurité réelle qu'ils ne seront plus jamais dépossédés.

La mémoire d'un événement traumatique et le patrimoine sont deux formes de rapports au passé, tous deux liés à des questions identitaires. À l'Anglo, l'histoire officielle s'arrête avec la fermeture de l'usine, puisque ce n'est qu'en fonctionnement qu'elle est le symbole du progrès. Le récit des habitants appelle pourtant à considérer une configuration plus complexe, une mémoire empêchée (Ricoeur, 2000), cachée ou mise en veille par une autre plus glorieuse, poussant à explorer ce que Jean-Michel Leniaud nomme «la stratification mémorielle d'un site» (Leniaud, 2003: 41). Comprenant la mémoire de l'usine et celle qui se construit après sa fermeture comme complémentaires plutôt que concurrentes, nous considérons également que « la justice descriptive contribue à la justice sociale » (Tornatore, 2007). La prise en compte de tous les discours, avec une égale légitimité, nous paraît être la condition d'une «politique de 
la juste mémoire » (Ricoeur, 2000), favorisant une relation apaisée avec le passé du quartier.

La fabrique du patrimoine et la mise aux normes qu'elle implique, le travail de la loi sur les lieux de vie occupés, relance un débat qui était comme endormi, ou qui se réglait dans l'entre soi du quartier, dans les relations ordinaires. Au-delà, ou en deçà des violations de droits humains perpétrés par la dictature militaire, face auxquelles l'État et les organisations cherchent à rendre justice, celle-ci s'est inscrite dans les villes. À travers différents projets urbains, aboutis ou non, le régime y a imprimé sa marque, de façon autoritaire ${ }^{21}$. Le traumatisme social qu'elle représente se trouve alors, aussi, dans ces «petites» affaires, dans une violence symbolique diffuse, touchant à différents aspects de la société. On comprend alors qu'aujourd'hui, tout projet patrimonial ou de réaménagement peut révéler, cristalliser ou réveiller des blessures, non « digérées », dont il faut bien faire quelque chose.

\section{Épilogue en images}

Cette mission au bord du fleuve Uruguay aura finalement soulevé essentiellement des questions et la volonté de mener plus avant notre recherche. Nos certitudes, à l'heure actuelle, sont peu nombreuses. La première est que la mémoire des anciens habitants de l'Anglo restera frustrée tant que l'on ne leur aura pas attribué des titres de propriété. On peut penser alors que le patrimoine immatériel de l'Anglo dépend de ce papier officiel, dans le sens où la mémoire du passé industriel serait comme suspendue, pour les habitants, par l'expectative de titularisation. Leur mémoire se focalise, en attendant, sur la dépossession. Les maisons sont bien le patrimoine, dans tous les sens du terme, des familles. La seconde est la nécessité éthique et historique «d'intégrer » les habitants du JC6 et du JC7 dans l'écriture du passé de l'Anglo.

\footnotetext{
21 À Montevideo un processus du même ordre existe actuellement. Dans les quartiers sud, en effet, plusieurs complexes d'habitations où vivaient principalement des Afro-uruguayens ont été détruits pendant la dictature, sous prétexte de vétusté. La population locale, expropriée, à été relogée dans des quartiers périphériques. Aujourd'hui, alors que le quartier est en cours de revalorisation et que des coopératives d'habitation sont en construction, la communauté afro-montévidéenne se mobilise, demandant à a fois le relogement de quelques familles déplacées et la reconnaissance de leur inscription culturelle dans ces « quartiers noirs » (Giorgiadis, 2009, Epstein, 2010).
} 
En nous centrant peu à peu sur le quartier et les lieux d'habitation, nous avons décidé de photographier les habitants que nous interrogions, chez eux ou sur leur perron.
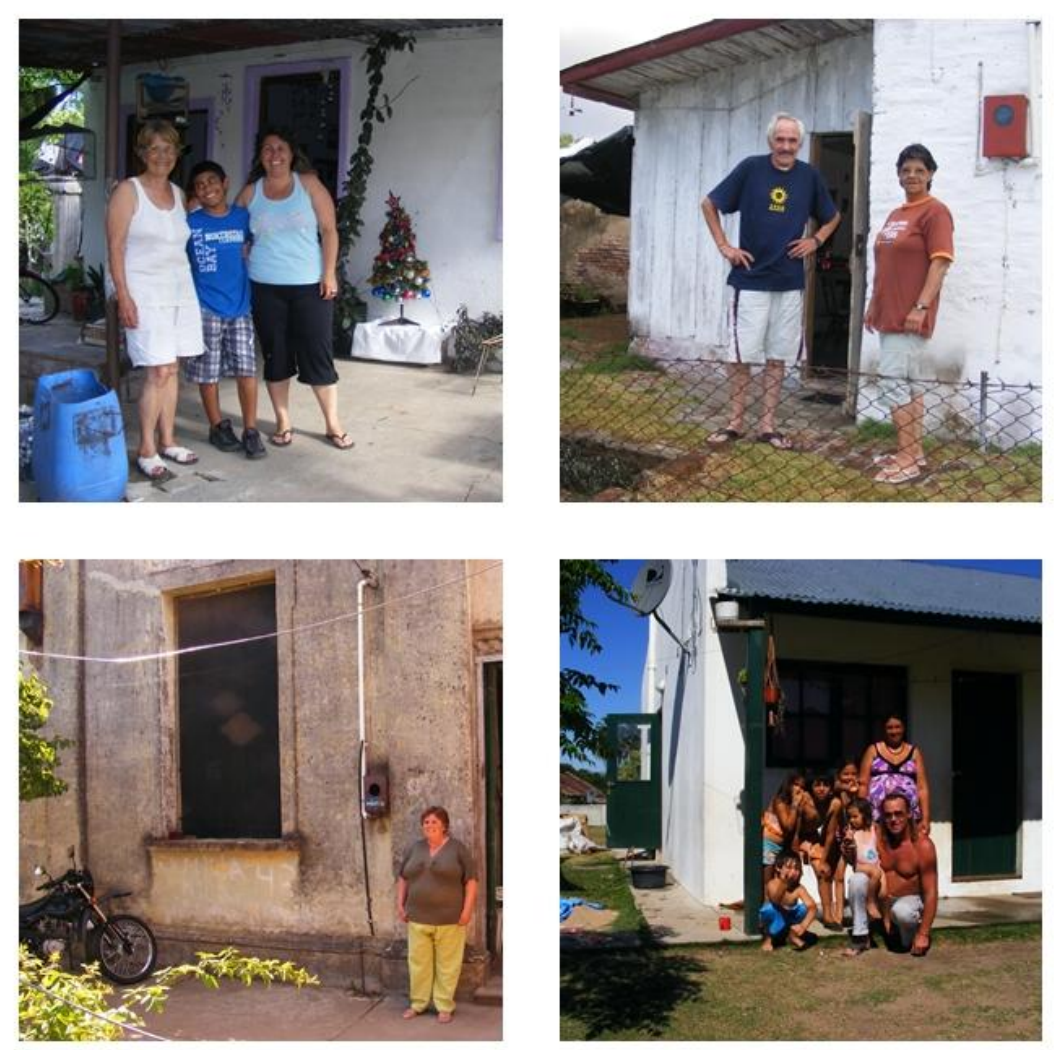

Photos 15, 16, 17, 18: Anciens, nouveaux et ex habitants de l'Anglo. Photographies Felipe Vargas.

Iluminuras, Porto Alegre, v. 14, n. 33, p. 100-124, jul./dez. 2013 

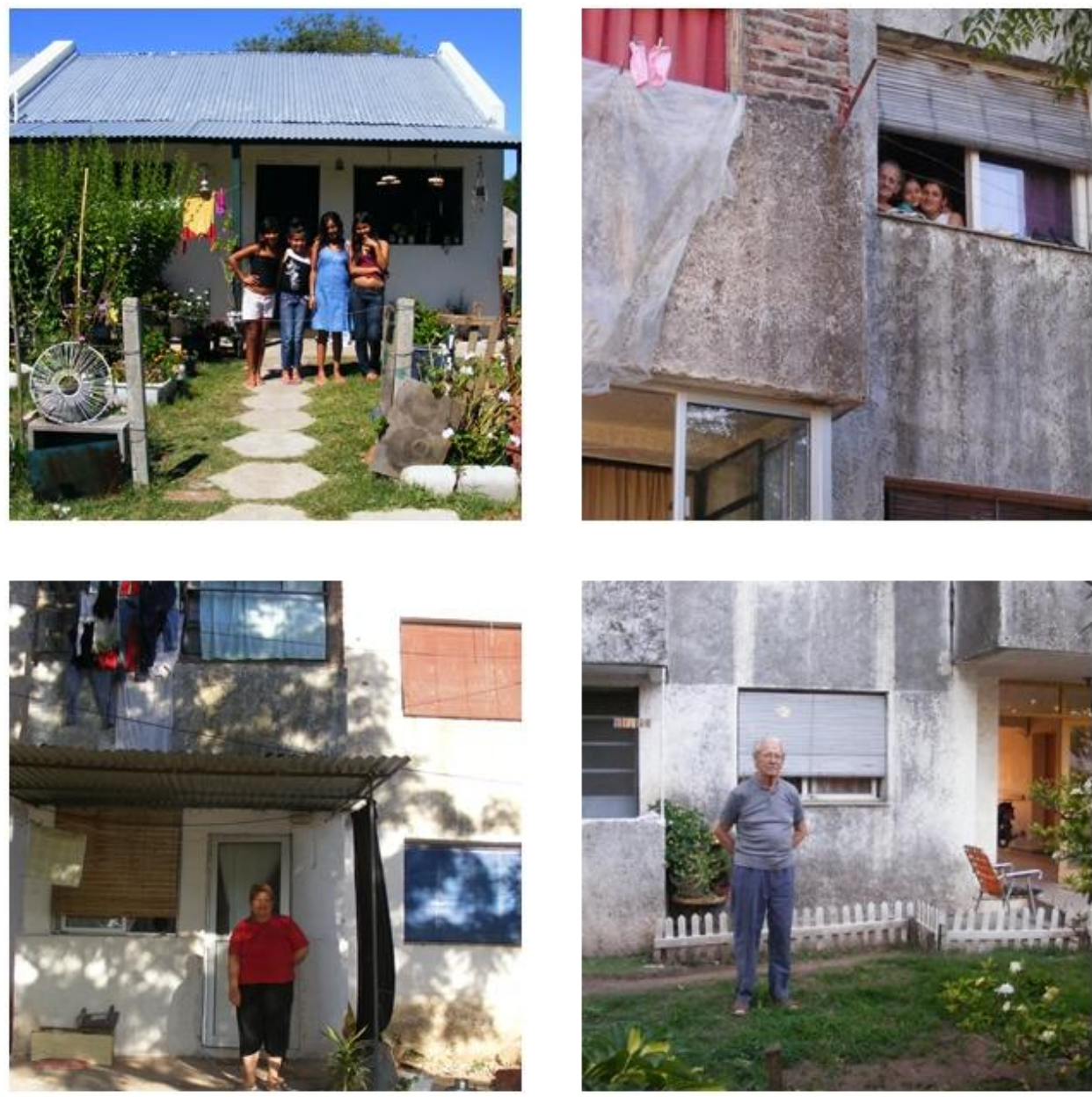

Photo 19, 20, 21, 22: Anciens, nouveaux et ex habitants de l'Anglo. Photographies Felipe Vargas.

Nous avons ainsi commencé à constituer une série d'images, qui se veut performative, un geste symbolique. En effet, chaque cliché est un objet tangible qui représente l'appartenance des maisons àleurs habitants et à l'inverse, celle des habitants à leur maison, et fait écho aux titres de propriété en débat. Ces images sont aussi une sorte de contre pied à un autre relevé de photographies, réalisé par les assistantes sociales du programme PIAI en 1992, qui montrait chacune des maisons du quartier, mais sans habitants ni aucun nom sur les légendes, les réduisant à leur seule dimension architecturale, ou immobilière. Montrant les différents habitats des anciens, nouveaux et ex-habitants de l'Anglo, il s'agit de donner à voir le groupe, pour le moins hétérogène, de ceux qui portent les mémoires de l'Anglo. Ce projet ethno-photographique, qui n'est 
qu'à sa première étape de réalisation ${ }^{22}$, vise donc à soulever de nouvelles questions, à proposer une manière d'impliquer les habitants dans le processus de patrimonialisation et de mise en récit de leur quartier, en les re-situant au centre de l'histoire.

\section{Références}

BENSA Alban et FASSIN Eric, 2002. «Les sciences sociales face à l'événement », Terrain $\mathrm{n}^{\circ}$ 38 , p. 5-20.

BORETTO OVALLE René, 2000. Historiografía de la ciudad de Fray Bentos (1857-1890), Fray Bentos, Ministerio de Educación y Cultura.

BORETTO OVALLE René, BURGUEÑO María Julia, "Perspectivas de uso turístico de los recursos patrimoniales de la industria de la carne en el Bajo Rio Uruguay", Presentado en el II Congreso Internacional sobre Patrimonio Industrial Agroalimentario (Córdoba, Argentina) 3-7 de noviembre de 2009, http://riouruguay.rionegrotodo.com/investigaciones-propias.

BORETTO OVALLE René, 1993. Antología de la apropiación indebida. La viveza criolla al servicio del hurto.Anecdotario del frigorífico ANGLO, Rabel, S.A. Mercedes.

BREVIGLIERI Marc, 2010. « De la cohésion de vie du migrant : déplacement migratoire et orientation existentielle », Revue européenne des migrations internationales [En ligne], vol. 26 $-\mathrm{n}^{\circ} 2$, http://remi.revues.org/index5137.html

CAMPODÓNICO Gabriela, 2000. «El frigorífico Anglo : Memoria urbana y memoria social en Fray Bentos", in in Anuario de Antropología social y cultural en Uruguay, Facultad de Humanidades y Ciencias de la Educación, Montevideo, p. 99-108.

CHAGAS Karla, GIORGIADIS Raquel, PASCUAL Cecilia, à paraître. « Patrimonio Inmaterial en la Comisión del Patrimonio Cultural de la Nación. Presentación y enfoques », Trama, $\mathrm{n}^{\circ} 4$, Montevideo.

DAUMAS Jean-Claude, 2006. «L'usine, la mémoire et l'histoire », Jean-Claude Daumas (dir.), La mémoire de l'industrie. De l'usine au patrimoine, Presses Universitaires de FrancheComté, p. 9-20.

EPSTEIN Ariela, 2010. «iArriba los que luchan!»Cultures politiques sur les murs de Montevideo, thèse de doctorat en anthropologie sociale et historique, sous la direction de Marlène Albert Llorca et Modesta Suárez, Université de Toulouse-Le Mirail.

FABRE Daniel, 2010. «introduction : habiter les monuments », FABRE Daniel, IUSO Anna (dir.) Les monuments sont habités, Paris, éditions de la maison des sciences de l'homme (collection cahiers d'ethnologie de la france, $\left.n^{\circ} 24\right)$, p. 17-52.

\footnotetext{
${ }^{22}$ Au-delà de la volonté de compléter ce recensement photographique, nous souhaitons aussi mettre en place des formes de restitution et de confrontation critique de notre travail aux habitants comme aux acteurs institutionnels.
}

Iluminuras, Porto Alegre, v. 14, n. 33, p. 100-124, jul./dez. 2013 
FABRE Daniel (ss.dir.), 2000. Domestiquer l'histoire : ethnologie des monuments historiques, Paris, Editions de la Maison des sciences de l'homme.

FASSIN Didier, RECHTMAN Richard, 2007. L'empire du traumatisme. Enquête sur la condition de victime, Paris, Flammarion, coll. Champs essais.

FESCHET Valérie et ISNART Cyril (coord.), 2013/1. "Pays perdus, pays imaginés », Ethnologie française, Vol. 43, Paris, PUF.

GIORGIADIS, Raquel, 2009. « Jaque a un monumento: construcciones y uso del patrimonio en el conventillo Anisna », Trama ${ }^{\circ} 1$, p. 66-81.

GUIBERT Martine, CARRIZO Silvina, LIGRONE Pablo, MALLARD Bruno, MÉNANTEAU Loïc et URIBE Guillermo (Ed.), 2009. Le Bassin du Río de la Plata : intégration régionale et développement local, Toulouse, Ed. des PUM, Coll. Hespérides Amérique.

LENIAUD Jean-Michel, 2003. "Vingt-cinq ans d'histoire du patrimoine », P. POIRRIER, L. VADELORGE, Pour une histoire des politiques du patrimoine, Travaux et Documents $\mathrm{n}^{\circ} 16$, Comité d'histoire du ministère de la culture, Paris, p. 33-44.

LOSONCZY Anne-Marie, 2006. «La muséification du passé récent en Hongrie postcommuniste », Revue d'étude comparatives Est-Ouest, vol. 37, n³, p. 97-112.

LUPANO María Marta, 2009. La gran familia industrial. Espacio Urbano, prácticas sociales e ideología (1870-1945), Santiago Arcos Editor, Buenos Aires.

MÉNANTEAU Loïc, BORETTO OVALLE René, 2005. «Le patrimoine culturel et industriel du Bas -Uruguay (Argentine, Uruguay) : typologie, spécificités et potentialités touristiques », travaux présenté lors de l'Université européenne d'été 2005 "Val de Loire - patrimoine mondial" Tours-Angers-Nantes 26 au 30 septembre 2005, http://riouruguay.rionegrotodo.com/investigaciones-propias

NORA Pierre, 1972. «L'événement monstre », Communications, n¹8, p. 162-172.

RAUTENBERG Michel, FAURAUT François, 1994. Patrimoine et culture industrielle, Lyon, CNRS.

REY ASHFIELD William, 2009. "Alternativas de uso y puesta en valor del patrimonio industrial: el caso de Fray Bentos", Patrimonio de la Nación,

« https://sites.google.com/site/sistemapatrimonial/investigaciones-1 »

RICOEUR Paul, 2000. La mémoire, l'histoire, l'oubli, Paris, Editions du Seuil, Points Seuil, Essais.

SCOTT James, 2009 [1999]. La Domination et les arts de la résistance. Fragments d'un discours subalterne, Paris, Editions Amsterdam. 
TAKS Javier, 2000. "La clase trabajadora y las obreras del ANGLO", Revista Encuentros, número 6, Montevideo, p. 211-230.

TORNATORE Jean-Louis, 2004. «La difficile politisation du patrimoine ethnologique », Terrain, ${ }^{\circ} 42$, p. $149-160$.

TORNATORE Jean-Louis, 2006. «Trou de mémoire. Une perspective post-industrielle de la «la Lorraine sidérurgique », Jean-Claude DAUMAS (dir.), La mémoire de l'industrie: de l'usine au patrimoine, Cahiers de la MSH Ladoux, Besançon, Presses Universitaires de FrancheComté, p. 49-80.

TORNATORE Jean-Louis, 2007. «Qu'est ce qu'un ethnologue politisé ? Expertise et engagement en socio-anthropologie de l'activité patrimoniale ». ethnographiques.org, Numéro 12 [en ligne]. http://www.ethnographiques.org

VESCHAMBRE Vincent, 2008. Traces et mémoires urbaines: enjeux sociaux de la patrimonialisation et de la démolition, Rennes, Presses Universitaires de Rennes.

Recebido em: 04/07/2013

Aprovado em: 29/07/2013 